\title{
After the ribosome structure: How does translocation work?
}

\author{
SIMPSON JOSEPH \\ Department of Chemistry and Biochemistry, University of California, San Diego, La Jolla, California 92093-0314, USA
}

\begin{abstract}
Structures of the ribosomal large and small subunits have been solved to atomic resolution by X-ray crystallography. These structures provide a new foundation to address the complex process of protein biosynthesis by the ribosome. Translocation of the tRNA-mRNA complex is one of the most fascinating tasks performed by the ribosome. The impact of the crystal structures in understanding the molecular mechanism of translocation is highlighted in this review.
\end{abstract}

As recently as the mid-1990s, obtaining high-resolution structure of the ribosome was considered to be an impossible dream. Today, it is feasible for anybody with a personal computer to download and view atomic resolution structures of both ribosomal subunits (Ban et al. 2000; Schluenzen et al. 2000, 2001; Wimberly et al. 2000). Additionally, structures of the complete ribosome incorporating tRNA and mRNA have been solved at $5.5-\AA$ resolution (Yusupov et al. 2001). This amazing change of events has provided a much-needed structural framework and has revolutionized the field. The remaining challenge is to understand the functional significance of the ribosome structure. One of the most remarkable events during the elongation phase of protein synthesis is the iterative movement of the tRNAs and the associated mRNA through the ribosome. The molecular basis for translocation and mRNA-reading-frame maintenance are largely unknown. Translocation is catalyzed by an elongation factor (EF-G in Escherichia coli) and involves precise and coordinated movement of large molecules (mRNA and two tRNAs) over long distances ( $\sim 50 \AA$ ) in the ribosome. Furthermore, the ribosome is a dynamic machine that undergoes dramatic conformational changes during translocation. Therefore, in this post-crystal-structure era, the most interesting challenge for ribosome research is to uncover the mechanism of translocation.

Reprint requests to: Simpson Joseph, 4102 Urey Hall, Department of Chemistry and Biochemistry, University of California, San Diego, 9500 Gilman Drive, La Jolla, CA 92093-0314, USA; e-mail: sjoseph@chem.ucsd. edu; fax: (858) 534-7042.

Article and publication are at http://www.rnajournal.org/cgi/doi/ 10.1261/rna.2163103.

\section{Models for translocation}

Ribosomes contain three tRNA-binding sites: the aminoacyl site (A site), the peptidyl site ( $\mathrm{P}$ site), and the exit site ( $\mathrm{E}$ site). Following peptide-bond formation, the ribosome has a deacylated tRNA in the $P$ site and a peptidyl tRNA in the A site (Fig. 1). The A site needs to be vacated in order to bind the next aminoacyl tRNA to the ribosome. According to the hybrid states model for translocation (Moazed and Noller 1989), movement of tRNA is proposed to occur in two steps: In the first step, which occurs spontaneously after the peptidyl transferase reaction, the acceptor ends of the deacylated tRNA and the peptidyl tRNA move relative to the large subunit from the $\mathrm{P}$ and $\mathrm{A}$ sites to the $\mathrm{E}$ and $\mathrm{P}$ sites, respectively, while their anticodon ends remain bound to the small subunit, resulting in $\mathrm{P} / \mathrm{E}$ and $\mathrm{A} / \mathrm{P}$ hybrid states (Fig. 1C). In the second step, which is EF-G-dependent, the anticodon arms of the two tRNAs move relative to the small subunit from the $\mathrm{P}$ and $\mathrm{A}$ sites to the $\mathrm{E}$ and $\mathrm{P}$ sites, respectively (Fig. 1D). At the end of translocation, deacylated tRNA is in the E site (E/E state), peptidyl tRNA is in the P site (P/P state), and EF-G-GDP dissociates from the ribosome.

Interestingly, the peptidyl tRNA in the $\mathrm{A} / \mathrm{P}$ hybrid state does not react with puromycin, an antibiotic analog of $\mathrm{A}$ site tRNA, indicating that it is functionally distinct from the $\mathrm{P} / \mathrm{P}$ state attained after translocation. Furthermore, an EF-G mutant lacking domain I (the GTP-binding domain) can partially translocate peptidyl tRNA from the A/P state to a puromycin-reactive state (Borowski et al. 1996). Thus, the spontaneous translocation of the acceptor ends of deacylated and peptidyl tRNA relative to the 50S large subunit is incomplete in the absence of EF-G. More recently, crystal- 


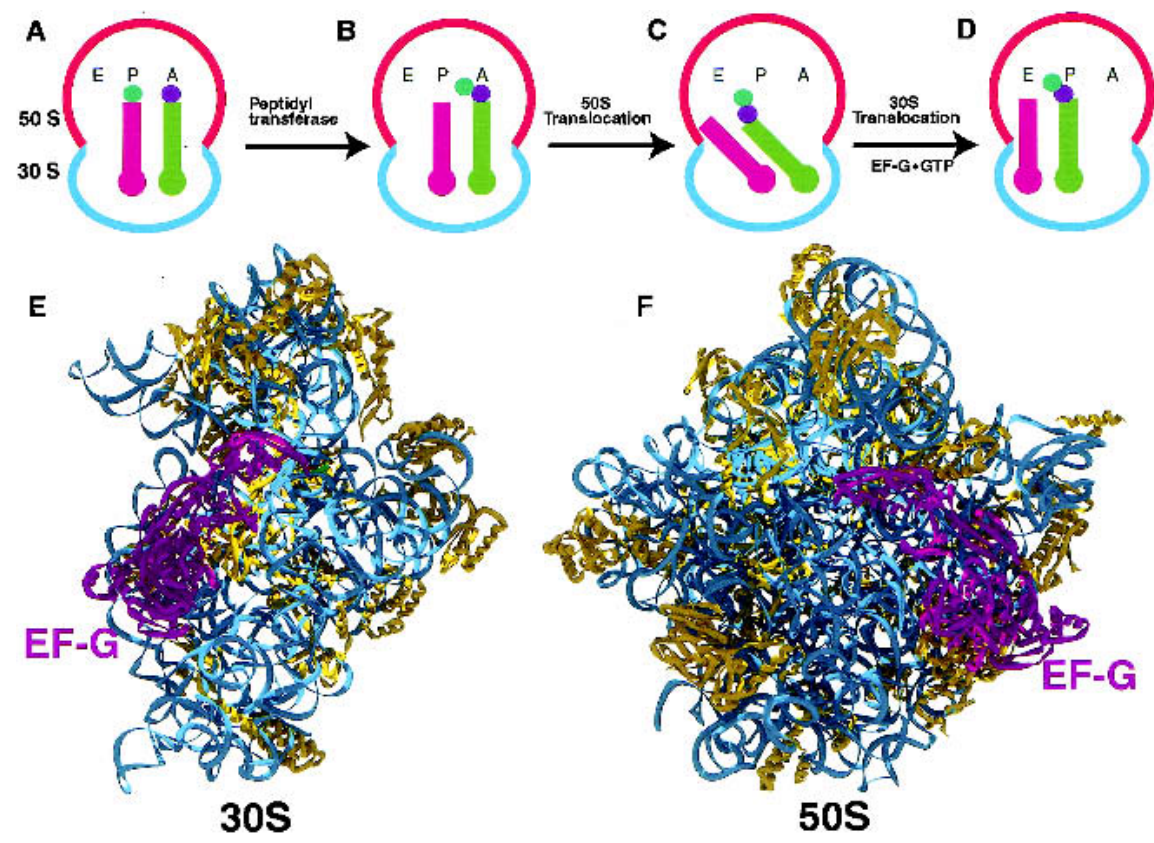

FIGURE 1. Revised hybrid states model for translocation (Moazed and Noller 1989). (A) Ribosome with a peptidyl tRNA in the P/P state (stem-loop structure in magenta) and an aminoacyl tRNA in the A/A state (stem-loop structure in green). Colored circles represent an amino acid attached to the 3 '-end of the tRNA. (B) After peptide-bond formation, deacylated tRNA is in the $\mathrm{P} / \mathrm{P}$ state and peptidyl tRNA extended by one amino acid is in the A/A state (Schmeing et al. 2002). $(C)$ The acceptor ends of the deacylated tRNA and the peptidyl tRNA are spontaneously translocated relative to the $50 \mathrm{~S}$ subunit, resulting in $\mathrm{P} / \mathrm{E}$ and $\mathrm{A} / \mathrm{P}$ hybrid states, respectively. $(D) \mathrm{EF}-\mathrm{G}$ moves the anticodon ends of the deacylated tRNA and peptidyl tRNA relative to the 30S subunit, resulting in E/E and P/P states, respectively. $(E, F)$ Approximate position of EF-G relative to the $30 \mathrm{~S}$ (Wimberly et al. 2000) and 50S (Ban et al. 2000) crystal structures viewed from the interface side. The docking of the EF-G crystal structure (AEvarsson et al. 1994) on the ribosomal subunits is based on data from directed hydroxyl radical probing (Wilson and Noller 1998a) and cryo-EM (Agrawal et al. 1998) studies.

lographic evidence has been obtained for another pretranslocation intermediate that contains the peptidyl tRNA ana$\log$ bound to the 50S-subunit A site after peptide-bond formation (Fig. 1B; Schmeing et al. 2002). Therefore, the peptidyl transferase reaction and the spontaneous $50 \mathrm{~S}$ translocation are not coupled but occur sequentially during the elongation cycle of protein synthesis. It is possible that there are additional transient intermediate states of translocation that remain to be discovered.

An alternative model for translocation called $\alpha-\epsilon$ was proposed by Nierhaus and colleagues (Dabrowski et al. 1998). They observed that the characteristic protection pattern of phosphorothioate-substituted tRNAs bound to the ribosomal $\mathrm{A}$ and $\mathrm{P}$ sites does not change upon translocation. This indicates that the tRNAs maintain their interactions with the respective ribosomal sites during translocation. Therefore, in addition to the swapping of contacts between tRNAs and the ribosomal sites during translocation as proposed by the hybrid states model, some of the contacts with tRNA may be preserved by a movable ribosomal domain that resets itself during A-site occupation. More studies are urgently needed to determine how this process is orchestrated by the ribosome.

\section{Interaction of tRNA and mRNA with the ribosome}

One of the immediate benefits of the crystal structures was the detailed information now available about ribosomal components that constitute the classical A, P, and E sites (for review, see Noller et al. 2001). The three tRNAs bind in the interface cavity, which is rich in rRNA elements from both the subunits. The anticodon arms of all three tRNAs bind to the 30 S subunit and interact with several $16 \mathrm{~S}$ rRNA residues and small subunit proteins. Interestingly, $16 \mathrm{~S}$ rRNA bases 530, 1492, and 1493, located in the 30S-subunit A site, undergo a conformational change to contact the codon-anticodon minihelix, providing new insights into the molecular basis for decoding (Ogle et al. 2001). The D-stem, elbow, and acceptor arm of $\mathrm{A}-, \mathrm{P}-$, and E-site tRNAs interact with $23 \mathrm{~S}$ rRNA and proteins present in the large subunit (Yusupov et al. 2001). The acceptor ends of A- and P-site tRNAs are close to each other and contact the peptidyl transferase region, which is, remarkably, composed exclusively of $23 \mathrm{~S}$ rRNA (Nissen et al. 2000). In contrast, the acceptor arm of the E-site tRNA is oriented away from the A- and P-site tRNAs and is close to the L1 stalk of the 50S subunit (Yusupov et al. 2001).

The mRNA loops around the head and shoulder of the 30 S subunit enclosing positions -15 to +15 within the ribosome (Yusupova et al. 2001). The Shine-Dalgarno sequence of the mRNA base-pairs with the complementary sequence present in the $3^{\prime}$-end of $16 \mathrm{~S}$ rRNA, forming a helix that is located between the head and the platform on the solvent side of the $30 \mathrm{~S}$ subunit. Positions -1 to +7 of the mRNA are exposed in the interface cavity, while the upstream and downstream sequence of the mRNA pass through tunnels formed by the head and shoulder of the small subunit. These tunnels may undergo conformational changes that permit the mRNA to move during translocation (Frank and Agrawal 2000; Schluenzen et al. 2000). Translocation of certain tRNAs can occur in the absence of mRNA template, indicating that mRNA-ribosome interactions are not critical for this process. However, during translocation, the tRNA-mRNA complex has to break contacts with the ribosome and establish a new set of interactions in the next site. This may be achieved in a step-wise fashion, wherein parts of the A- and P-site tRNAs break 
their interaction in one site and form new contacts in the next site.

Consistent with this idea, modifications to the 3 '-acceptor arm of the P-site tRNA that reduce E-site affinity also inhibit translocation (Lill et al. 1989). Ribose 2'-hydroxyl groups at positions 71 and 76 of P-site tRNA are important for translocation (Feinberg and Joseph 2001). These 2'hydroxyl groups contact $23 \mathrm{~S}$ rRNA elements in the 50Ssubunit E site (Yusupov et al. 2001), which indicates that movement of the acceptor arm of P-site tRNA into the large-subunit $\mathrm{E}$ site is a prerequisite for EF-G-dependent translocation of the anticodon arms relative to the small subunit.

Surprisingly, an anticodon stem-loop analog of tRNA (ASL) is translocated from the ribosomal A site, implying that interactions between the ribosome and the D-stem, elbow, and acceptor stem of A-site tRNA are not critical for translocation (Joseph and Noller 1998). In the absence of an ASL in the A site, the P-site tRNA and the associated mRNA are not translocated, indicating that interactions involving the ASL and the 30S-subunit A site play an essential role in translocation. Interestingly, the ribose 2'-hydroxyl groups at positions 33, 35, and 36 in the A-site ASL are important for translocation (Phelps et al. 2002). The ribose 2 '-hydroxyl groups at positions 35 and 36 contact the universally conserved 16S rRNA bases G530, A1492, and A1493, indicating that the fundamental mechanism for decoding and translocation may be related and similar in all organisms.

However, the translocation rate of an A-site-bound ASL is considerably slower compared with peptidyl tRNA, the physiological substrate (S. Studer, J. Feinberg, and S. Joseph, unpubl.). Therefore, interactions between the D-stem, elbow, and acceptor stem of A-site tRNA and the ribosome are likely to play a role in increasing the efficiency of translocation. Indeed, peptidyl tRNA with an A76G mutation in the 3 '-CCA end inhibits translocation from the A site to the $\mathrm{P}$ site, demonstrating the importance of the rest of the peptidyl tRNA for translocation (Virumae et al. 2002).

\section{Interaction of EF-G with the ribosome}

Elongation factor G (EF-G) consists of five structural domains (AEvarsson et al. 1994; Czworkowski et al. 1994), and it shows striking similarity with the elongation factor $\mathrm{Tu}$ $($ EF-Tu $) \cdot$ GTP-tRNA ternary complex in overall shape and charge distribution (Nissen et al. 1995). EF-G interacts with the 50S subunit near the base of the L7/L12 stalk and with the sarcin-ricin stem-loop region of 23S rRNA (Skold 1983; Moazed et al. 1988). Both these regions are close to each other in three dimensions in the crystal structures. EF-Tu (Moazed et al. 1988) and initiation factor 2 (IF2; Cameron et al. 2002) also protect nucleotides in the sarcin-ricin stem-loop region, which is consistent with the idea that these factors interact in a similar fashion with the ribosome.
Directed hydroxyl radical probing (Wilson and Noller 1998a) and independent cryo-EM studies (Agrawal et al. 1998) of EF-G - GDP stabilized on the ribosome with fusidic acid placed EF-G in the interface cavity with domain 5 of EF-G close to the base of the L7/L12 stalk, whereas domain 1 ( $G$ domain) contacts the sarcin-ricin region (Fig. $1 \mathrm{E}, \mathrm{F})$. Interestingly, domain 4 of EF-G reaches into the decoding region of the $30 \mathrm{~S}$ subunit. The position of EF-G in the ribosome is similar to the location of the EF-Tu ternary complex observed by cryo-EM (Stark et al. 1997). The G domain of EF-Tu is near the sarcin-ricin region of the ribosome, and the anticodon arm of tRNA in the ternary complex is in the $30 \mathrm{~S}$ decoding region. The similarity in size, shape, and orientation on the ribosome (especially of domain 4 of EF-G and the anticodon arm of tRNA in the ternary complex) has fueled speculation that the two factors may use a similar mechanism to perform different tasks on the ribosome.

Pre-steady-state kinetics analysis showed that GTP-hydrolysis by EF-G precedes and greatly accelerates translocation (Rodnina et al. 1997). The molecular mechanism for coupling the energy released from GTP hydrolysis to mechanical movement is unclear. Remarkably, ribosomes can translocate the tRNA-mRNA complex at a slow rate even in the absence of EF-G and GTP-hydrolysis (for review, see Spirin 1985). This implies that EF-G merely acts as a catalyst and that all the components for translocation are already present within the ribosome.

Quantitative kinetics analysis of binding and of GTPhydrolysis by wild type and EF-G mutants has provided valuable information about the functional steps in translocation. Mutation of a single amino acid at position 583 in the tip of domain 4 of EF-G inhibits translocation by $>100$ fold without affecting factor binding, GTP-hydrolysis, and release of inorganic phosphate $\left(\mathrm{PP}_{\mathrm{i}}\right.$; Savelsbergh et al. 2000). Similarly, deletion of domain 4 from EF-G causes a 1000 -fold reduction in translocation rate, without affecting factor binding or GTPase activity in the ribosome (Rodnina et al. 1997). Domain 4 in EF-G may couple GTP-hydrolysis to tRNA movement. An EF-G mutant that was conformationally restricted by an engineered disulfide bond between domains 1 and 5 shows $\sim 10^{4}$-fold inhibition in translocation, indicating that conformational flexibility of EF-G is important for translocation (Peske et al. 2000).

\section{Inhibition of translocation}

Antibiotic inhibitors of translocation may work by preventing EF-G or the ribosome (see below) from undergoing structural changes. Viomycin, a potent inhibitor of translocation, binds between the two subunits (Moazed and Noller 1987; Powers and Noller 1994) and may block the relative movement of the two subunits. Thiostrepton binds to the 1060 region of $23 \mathrm{~S}$ rRNA near the base of the L7/L12 stalk in the large subunit (Rosendahl and Douthwaite 
1994). The L7/L12 stalk is thought to undergo conformational changes that are important for translocation (Agrawal et al. 1999). Because EF-G also binds to the same region of 23S rRNA, it is possible that thiostrepton stabilizes a conformation in $23 \mathrm{~S}$ rRNA that inhibits EF-G binding (Cameron et al. 2002) or tRNA movement (Rodnina et al. 1999). In contrast to the above two inhibitors, fusidic acid allows EF-G-dependent GTP-hydrolysis and tRNA movement but blocks the dissociation of EF-G-GDP from the ribosome. Complexes of the translocation inhibitor spectinomycin or hygromycin B with the 30 S subunit have been solved by X-ray crystallography. Spectinomycin binds to the minor groove of helix 34 of $16 \mathrm{~S}$ rRNA and may block conformational changes in the $30 \mathrm{~S}$ head region that are important for translocation (Carter et al. 2000). Hygromycin B binds to the upper part of helix 44 in 16S rRNA (Brodersen et al. 2000), a region that has been implicated to move during translocation (Frank and Agrawal 2000; VanLoock et al. 2000). Hygromycin B may inhibit translocation by preventing movement of helix 44. Understanding the mechanism of translocation inhibitors may help in the rational design of novel antibiotics that target the ribosome.

\section{Ribosome dynamics during translocation}

The ribosome is not static but undergoes large-scale conformational changes during translocation (for review, see Wilson and Noller 1998b). Small-angle neutron scattering studies showed that the shape of the $30 \mathrm{~S}$ subunit changes and the intersubunit distance increase upon translocation (Serdyuk et al. 1992). In addition, conformational changes in the 70S are triggered by a switch in helix 27 of $16 \mathrm{~S}$ rRNA, thereby affecting the accuracy of translation (Lodmell and Dahlberg 1997; Gabashvili et al. 1999). This conformational switch may also play a role during translocation. Lead cleavage experiments indicate that the peptidyl transferase region in $23 \mathrm{~S}$ rRNA undergoes periodic structural changes during translocation (Polacek et al. 2000). More recently, tantalizing clues to the nature of these changes were obtained from cryo-EM studies. Ribosomal complexes with EF-G stably bound using GTP analogs, thiostrepton or fusidic acid, were analyzed and conformational changes were observed in EF-G and in both the large and small subunits (Frank and Agrawal 2000; Stark et al. 2000). The two subunits undergo a ratchet-like movement relative to each other during translocation. Changes were observed close to the mRNA channel, in the upper part of helix 44 in 16S rRNA, and in the intersubunit bridges (B1a, B1b, and B1c) that connect the $30 \mathrm{~S}$ subunit head with the $50 \mathrm{~S}$ subunit (Frank and Agrawal 2000). The role of ribosomal proteins in translocation must not be overlooked. Some of the intersubunit bridges are composed of ribosomal protein, and they also interact with tRNA and mRNA (Yusupov et al. 2001). The intersubunit bridges may play an important role during translocation by coordinating the movement of the tRNAs relative to the two subunits.

Clearly, high-resolution structures of the intermediate steps are essential for understanding the mechanism of translocation. In the meantime, available crystal structures will serve as the framework for designing and interpreting biochemical and genetic experiments that are focused on the functional characterization of this complex process. Combining high-resolution crystallographic data with cryoEM studies and molecular dynamics simulation will also prove powerful in understanding ribosome dynamics during translocation. Now that we have joyfully peered under the hood of this exquisite molecular machine, the exciting new frontier of understanding the structural basis of translocation awaits.

\section{ACKNOWLEDGMENTS}

I thank all the members of my lab for comments on this manuscript. This work was supported by grants from the NSF, NIH, and HFSP.

\section{REFERENCES}

AEvarsson, A., Brazhnikov, E., Garber, M., Zheltonosova, J., Chirgadze, Y., al-Karadaghi, S., Svensson, L.A., and Liljas, A. 1994. Three-dimensional structure of the ribosomal translocase: Elongation factor G from Thermus thermophilus. EMBO J. 13: 3669-3677.

Agrawal, R.K., Penczek, P., Grassucci, R.A., and Frank, J. 1998. Visualization of elongation factor $\mathrm{G}$ on the Escherichia coli $70 \mathrm{~S}$ ribosome: The mechanism of translocation. Proc. Natl. Acad. Sci. 95: 6134-6138.

Agrawal, R.K., Heagle, A.B., Penczek, P., Grassucci, R.A., and Frank, J. 1999. EF-G-dependent GTP hydrolysis induces translocation accompanied by large conformational changes in the $70 \mathrm{~S}$ ribosome. Nat. Struct. Biol. 6: 643-647.

Ban, N., Nissen, P., Hansen, J., Moore, P.B., and Steitz, T.A. 2000. The complete atomic structure of the large ribosomal subunit at $2.4 \AA$ resolution. Science 289: 905-920.

Borowski, C., Rodnina, M.V., and Wintermeyer, W. 1996. Truncated elongation factor $\mathrm{G}$ lacking the $\mathrm{G}$ domain promotes translocation of the $3^{\prime}$ end but not of the anticodon domain of peptidyl-tRNA. Proc. Natl. Acad. Sci. 93: 4202-4206.

Brodersen, D.E., Clemons Jr., W.M., Carter, A.P., Morgan-Warren, R.J., Wimberly, B.T., and Ramakrishnan, V. 2000. The structural basis for the action of the antibiotics tetracycline, pactamycin, and hygromycin B on the $30 \mathrm{~S}$ ribosomal subunit. Cell 103: 1143-1154.

Cameron, D.M., Thompson, J., March, P.E., and Dahlberg, A.E. 2002. Initiation factor IF2, thiostrepton and micrococcin prevent the binding of elongation factor $\mathrm{G}$ to the Escherichia coli ribosome. J. Mol. Biol. 319: 27-35.

Carter, A.P., Clemons, W.M., Brodersen, D.E., Morgan-Warren, R.J., Wimberly, B.T., and Ramakrishnan, V. 2000. Functional insights from the structure of the $30 \mathrm{~S}$ ribosomal subunit and its interactions with antibiotics. Nature 407: 340-348.

Czworkowski, J., Wang, J., Steitz, T.A., and Moore, P.B. 1994. The crystal structure of elongation factor G complexed with GDP, at $2.7 \AA$ resolution. EMBO J. 13: 3661-3668.

Dabrowski, M., Spahn, C.M., Schafer, M.A., Patzke, S., and Nierhaus, K.H. 1998. Protection patterns of tRNAs do not change during ribosomal translocation. J. Biol. Chem. 273: 32793-32800.

Feinberg, J.S. and Joseph, S. 2001. Identification of molecular interactions between P site tRNA and the ribosome essential for translocation. Proc. Natl. Acad. Sci. 98: 11120-11125. 
Frank, J. and Agrawal, R.K. 2000. A ratchet-like inter-subunit reorganization of the ribosome during translocation. Nature 406: 318-322.

Gabashvili, I.S., Agrawal, R.K., Grassucci, R., Squires, C.L., Dahlberg, A.E., and Frank, J. 1999. Major rearrangements in the 70S ribosomal 3D structure caused by a conformational switch in $16 \mathrm{~S}$ ribosomal RNA. EMBO J. 18: 6501-6507.

Joseph, S. and Noller, H.F. 1998. EF-G-catalyzed translocation of anticodon stem-loop analogs of transfer RNA in the ribosome. EMBO J. 17: 3478-3483.

Lill, R., Robertson, J.M., and Wintermeyer, W. 1989. Binding of the $3^{\prime}$ terminus of tRNA to $23 \mathrm{~S}$ rRNA in the ribosomal exit site actively promotes translocation. EMBO J. 8: 3933-3938.

Lodmell, J.S. and Dahlberg, A.E. 1997. A conformational switch in Escherichia coli $16 \mathrm{~S}$ ribosomal RNA during decoding of messenger RNA. Science 277: 1262-1267.

Moazed, D. and Noller, H.F. 1987. Chloramphenicol, erythromycin, carbomycin and vernamycin B protect overlapping sites in the peptidyl transferase region of $23 \mathrm{~S}$ ribosomal RNA. Biochimie 69: $879-884$.

. 1989. Intermediate states in the movement of transfer RNA in the ribosome. Nature 342: 142-148.

Moazed, D., Robertson, J.M., and Noller, H.F. 1988. Interaction of elongation factors EF-G and EF-Tu with a conserved loop in $23 \mathrm{~S}$ RNA. Nature 334: 362-364.

Nissen, P., Kjeldgaard, M., Thirup, S., Polekhina, G., Reshetnikova, L., Clark, B.F.C., and Nyborg, J. 1995. Crystal structure of the ternary complex of Phe-tRNA ${ }^{\text {Phe }}$, EF-Tu, and a GTP analog. Science 270: 1464-1472.

Nissen, P., Hansen, J., Ban, N., Moore, P.B., and Steitz, T.A. 2000. The structural basis of ribosome activity in peptide bond synthesis. Science 289: 920-930.

Noller, H.F., Yusupov, M.M., Yusupova, G.Z., Baucom, A., Lieberman, K., Lancaster, L., Dallas, A., Fredrick, K., Earnest, T.N., and Cate, J.H.D. 2001. Structure of the ribosome at $5.5 \AA$ resolution and its interactions with functional ligands. In The ribosome (Cold Spring Harbor Symposia on Quantitative Biology), pp. 57-66. Cold Spring Harbor Laboratory Press, Cold Spring Harbor, NY.

Ogle, J.M., Brodersen, D.E., Clemons Jr., W.M., Tarry, M.J., Carter, A.P., and Ramakrishnan, V. 2001. Recognition of cognate transfer RNA by the 30S Ribosomal subunit. Science 292: 897-902.

Peske, F., Matassova, N.B., Savelsbergh, A., Rodnina, M.V., and Wintermeyer, W. 2000. Conformationally restricted elongation factor $\mathrm{G}$ retains GTPase activity but is inactive in translocation on the ribosome. Mol. Cell 6: 501-505.

Phelps, S.S., Jerinic, O., and Joseph, S. 2002. Universally conserved interactions between the ribosome and the anticodon stem-loop of A site tRNA important for translocation. Mol. Cell 10: 799-807.

Polacek, N., Patzke, S., Nierhaus, K.H., and Barta, A. 2000. Periodic conformational changes in rRNA: Monitoring the dynamics of translating ribosomes. Mol. Cell 6: 159-171.

Powers, T. and Noller, H.F. 1994. Selective perturbation of G530 of 16 $S$ rRNA by translational miscoding agents and a streptomycindependence mutation in protein S12. J. Mol. Biol. 235: 156-172.

Rodnina, M.V., Savelsbergh, A., Katunin, V.I., and Wintermeyer, W. 1997. Hydrolysis of GTP by elongation factor G drives tRNA movement on the ribosome. Nature 385: 37-41.

Rodnina, M.V., Savelsbergh, A., Matassova, N.B., Katunin, V.I., Semenkov, Y.P., and Wintermeyer, W. 1999. Thiostrepton inhibits the turnover but not the GTPase of elongation factor G on the ribosome. Proc. Natl. Acad. Sci. 96: 9586-9590.

Rosendahl, G. and Douthwaite, S. 1994. The antibiotics micrococcin and thiostrepton interact directly with $23 \mathrm{~S}$ rRNA nucleotides 1067A and 1095A. Nucleic Acids Res. 22: 357-363.

Savelsbergh, A., Matassova, N.B., Rodnina, M.V., and Wintermeyer, W. 2000. Role of domains 4 and 5 in elongation factor $\mathrm{G}$ functions on the ribosome. J. Mol. Biol. 300: 951-961.

Schluenzen, F., Tocilj, A., Zarivach, R., Harms, J., Gluehmann, M., Janell, D., Bashan, A., Bartels, H., Agmon, I., Franceschi, F., et al. 2000. Structure of functionally activated small ribosomal subunit at 3.3 angstroms resolution. Cell 102: 615-623.

Schlunzen, F., Zarivach, R., Harms, J., Bashan, A., Tocilj, A., Albrecht, R., Yonath, A., and Franceschi, F. 2001. Structural basis for the interaction of antibiotics with the peptidyl transferase centre in eubacteria. Nature 413: 814-821.

Schmeing, T.M., Seila, A.C., Hansen, J.L., Freeborn, B., Soukup, J.K., Scaringe, S.A., Strobel, S.A., Moore, P.B., and Steitz, T.A. 2002. A pre-translocational intermediate in protein synthesis observed in crystals of enzymatically active 50S subunits. Nat. Struct. Biol. 9: $225-230$.

Serdyuk, I., Baranov, V., Tsalkova, T., Gulyamova, D., Pavlov, M., Spirin, A., and May, R. 1992. Structural dynamics of translating ribosomes. Biochimie 74: 299-306.

Skold, S.E. 1983. Chemical crosslinking of elongation factor $G$ to the 23S RNA in 70S ribosomes from Escherichia coli. Nucleic Acids Res. 11: 4923-4932.

Spirin, A.S. 1985. Ribosomal translocation: Facts and models. Prog. Nucleic Acid Res. Mol. Biol. 32: 75-114.

Stark, H., Rodnina, M.V., Rinke-Appel, J., Brimacombe, R., Wintermeyer, W., and van Heel, M. 1997. Visualization of elongation factor Tu on the Escherichia coli ribosome. Nature 389: 403-406.

Stark, H., Rodnina, M.V., Wieden, H.J., van Heel, M., and Wintermeyer, W. 2000. Large-scale movement of elongation factor $G$ and extensive conformational change of the ribosome during translocation. Cell 100: 301-309.

VanLoock, M.S., Agrawal, R.K., Gabashvili, I.S., Qi, L., Frank, J., and Harvey, S.C. 2000. Movement of the decoding region of the $16 \mathrm{~S}$ ribosomal RNA accompanies tRNA translocation. J. Mol. Biol. 304: $507-515$.

Virumae, K., Saarma, U., Horowitz, J., and Remme, J. 2002. Functional importance of the $3^{\prime}$-terminal adenosine of tRNA in ribosomal translation. J. Biol. Chem. 277: 24128-24134.

Wilson, K.S. and Noller, H.F. 1998a. Mapping the position of translational elongation factor EF-G in the ribosome by directed hydroxyl radical probing. Cell 92: 131-139.

. 1998b. Molecular movement inside the translational engine. Cell 92: 337-349.

Wimberly, B.T., Brodersen, D.E., Clemons Jr., W.M., Morgan-Warren, R.J., Carter, A.P., Vonrhein, C., Hartsch, T., and Ramakrishnan, V. 2000. Structure of the $30 \mathrm{~S}$ ribosomal subunit. $\mathrm{Na}$ ture 407: 327-339.

Yusupov, M.M., Yusupova, G.Z., Baucom, A., Lieberman, K., Earnest, T.N., Cate, J.H.D., and Noller, H.F. 2001. Crystal structure of the ribosome at $5.5 \AA$ resolution. Science 292: 883-896.

Yusupova, G.Z., Yusupov, M.M., Cate, J.H., and Noller, H.F. 2001. The path of messenger RNA through the ribosome. Cell 106: 233241. 

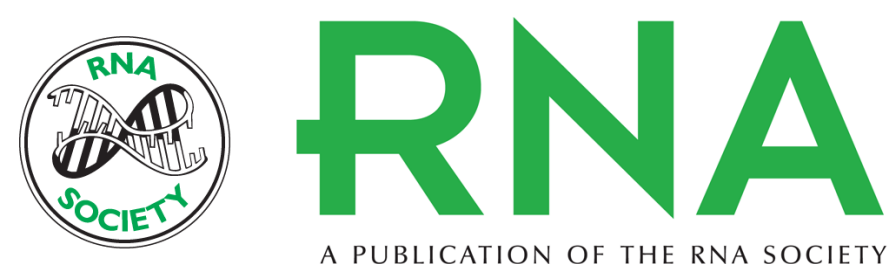

A PUBLICATION OF THE RNA SOCIETY

\section{After the ribosome structure: How does translocation work?}

\section{SIMPSON JOSEPH}

RNA 2003 9: 160-164

$\begin{array}{ll}\text { References } & \begin{array}{l}\text { This article cites } 44 \text { articles, } 13 \text { of which can be accessed free at: } \\ \text { http://rnajournal.cshlp.org/content/9/2/160.full.html\#ref-list-1 }\end{array}\end{array}$

License

Email Alerting Receive free email alerts when new articles cite this article - sign up in the box at the Service top right corner of the article or click here.

To subscribe to RNA go to:

http://rnajournal.cshlp.org/subscriptions 